\title{
A recurrent, fatal, DRESS syndrome, complicated by sepsis and severe systemic cytomegalovirus reactivation at relapse: a case report
}

\author{
Anna Castrovilli, ${ }^{1}$ Tommaso Mastrofilippo, ${ }^{1}$ Lucia Di Fino, ${ }^{1}$ Caterina Foti, ${ }^{2}$ Giuseppe Ingravallo, ${ }^{3}$ Antonio Perrone, ${ }^{1}$ \\ Carlo Sabbà, ${ }^{1}$ Francesco Saverio Vella ${ }^{1}$ \\ ${ }^{1}$ Interdisciplinary Department of Medicine, University Hospital Azienda Ospedaliera Consorziale Policlinico, Internal Medicine \\ Unit Cesare Frugoni, School of Medicine, University of Bari; ${ }^{2}$ Department of Internal Medicine, Immunology and Infectious \\ Diseases, Dermatology Clinic, School of Medicine, University of Bari; ${ }^{3}$ Department of Hematology and Organ Transplantation, \\ University Hospital Azienda Ospedaliera Consorziale Policlinico, School of Medicine, University of Bari, Italy
}

\begin{abstract}
We report a case of recurrent, fatal, DRESS (drug reaction with eosinophilia and systemic symptoms) syndrome induced by allopurinol. A 57-year-old man was admitted to our clinic manifesting a severe skin rash and fever, after following a four-weeklong allopurinol therapy. On the basis of clinical, laboratory and instrumental features, the patient was diagnosed with DRESS syndrome, following RegiSCAR and Japanese group's criteria. The clinical course of the disease was complicated by viral infection, methicillin-resistant Staphylococcus aureus endocarditis, bacterial pneumonia and severe recurrences. Despite allopurinol therapy was suspended and systemic steroids, targeted antibiotics, ganciclovir and immunoglobulins were administered, the clinical course had a fatal outcome. Herpes viruses have shown to have a crucial role in the pathogenesis of severe DRESS syndrome, suggesting that an early use of antiviral therapy, in addition to steroids, could improve the prognosis of DRESS syndrome.
\end{abstract}

\section{Case Report}

In December 2016, a 57-year-old Italian Caucasian man suddenly developed a diffuse skin rash reaction, accompanied by fever, dyspnea and productive cough.

His past history was remarkable considering that he

Correspondence: Anna Castrovilli, Interdisciplinary Department of Medicine, University Hospital Azienda Ospedaliera Consorziale Policlinico, Internal Medicine Unit Cesare Frugoni, School of Medicine, University of Bari, Piazza G. Cesare 11, 70100 Bari, Italy.

E-mail: annacastrovilli@me.com

Key words: DRESS syndrome; allopurinol; Herpes viruses; sepsis; antivirals.

Conflict of interest: the authors declare no potential conflict of interest.

Received for publication: 21 September 2018.

Revision received: 25 October 2018.

Accepted for publication: 25 October 2018.

This work is licensed under a Creative Commons Attribution NonCommercial 4.0 License (CC BY-NC 4.0).

CCopyright A. Castrovilli et al., 2018

Licensee PAGEPress, Italy

Italian Journal of Medicine 2019; 13:48-53

doi:10.4081/itjm.2018.1091 was affected by rheumatic endocarditis in his twenties and was subject to aortic and mitral replacement with mechanical valves at 31 years of age. He was later diagnosed with severe dilated cardiomyopathy (ejection fraction 20\%) and permanent atrial fibrillation with subsequent insertion of implantable cardioverterdefibrillator (ICD) at 46 years of age, which was removed and re-implanted at another site, one year later, due to pulse generator pocket infection. He reported a marked weight loss $(12 \mathrm{~kg})$ in recent times (last 6 months) and was diagnosed with asymptomatic hyperuricemia one month before admission, during a routine clinical follow-up, which was treated by allopurinol therapy $(150 \mathrm{mg} /$ daily). The oral therapy chronically administered to the patient consisted in: furosemide (25 mg twice-daily), bisoprolol (5 mg twice-daily), ranitidine (300 md once-daily) and warfarin (depending on prothrombin time/international normalized ratio).

At admission to our clinic the patient presented slight confusion (GCS 14$)$, fever $\left(39^{\circ} \mathrm{C}\right)$, a maculopapular-desquamative erythroderma, peri-nasal and peri-oral Herpes-like lesions, conjunctival burning, facial and generalized edema, onychodystrophy and lymphadenopathy (Figure 1). Joint pain was absent. He had an arrhythmic pulse at $102 \mathrm{rpm}$, blood pressure at $90 / 60 \mathrm{mmHg}$, respiratory rate at $40 \mathrm{bpm}$, bilateral vesicular murmur reduction and diffuse rhonchi. On the same day of admission, an acute cardiac arrest was 
promptly resolved by ICD without the need of cardiopulmonary resuscitation.

Laboratory data revealed anemia, leukocytosis with eosinophilia, thrombocytopenia and altered hepatic, pancreatic and renal function, as suggested by the following parameters: hemoglobin $10.6 \mathrm{~g} / \mathrm{dL}$, white blood cells count $12.7 \times 10^{3} / \mathrm{mm}^{3}$ (neutrophils: $35.4 \%$, lymphocytes: $37.6 \%$, eosinophils: $21.2 \%-2692 / \mathrm{mm}^{3}$ ), platelets $74 \times 10^{3} / \mathrm{mm}^{3}$, T-lymphocyte subpopulation anomalies (inversion of CD4/CD8 ratio), creatinine 1.7 $\mathrm{mg} / \mathrm{dL}$, estimated glomerular filtration rate $43 \mathrm{~mL} / \mathrm{min}$, alanine aminotransferase $235 \mathrm{U} / \mathrm{L}$, aspartate aminotransferase $135 \mathrm{U} / \mathrm{L}, \quad$ gamma-glutamyl transpeptidase $195 \mathrm{U} / \mathrm{L}$, alkaline phosphatase $197 \mathrm{U} / \mathrm{L}$, pancreatic amylase $404 \mathrm{U} / \mathrm{L}$, lipase $488 \mathrm{U} / \mathrm{L}$, C-reactive protein $90 \mathrm{mg} / \mathrm{L}$, erythrocyte sedimentation rate 78 $\mathrm{mm} / \mathrm{h}$, S-NT-proBNP 34,344 pg/mL, abnormal arterialblood gas test $\left(\mathrm{pO}_{2} 60 \mathrm{mmHg}, \mathrm{pCO}_{2} 28.6 \mathrm{mmHg}, \mathrm{pH}\right.$ 7.30 , lactic acid $5.3 \mathrm{mmol} / \mathrm{L}$ and $\mathrm{HCO}_{3}{ }^{-} 21.5 \mathrm{mmol} / \mathrm{L}$ ), and cardiac Troponin-I $0.184 \mathrm{ng} / \mathrm{mL}$. However, electrophoresis, serum Ig G-A-M, serum neoplastic markers (carcinoembryonic antigen $2.8 \mathrm{ng} / \mathrm{mL}$, alpha fetoprotein $2.7 \mathrm{ng} / \mathrm{mL}$, carbohydrate antigen-19.9 8.3 $\mathrm{U} / \mathrm{mL}$, total-prostate specific antigen $0.71 \mathrm{ng} / \mathrm{mL}$ ), and urinalysis were normal.

HLA phenotyping presented the following pattern: HLA-A1, -A2, -B17, -B51, -Bw4, -Cw6, -DR7, DR15, -DRw51, -DQ1, -DQ9. Nail mycological examination resulted negative. No evidence of antinuclear, anti-double stranded DNA, antidesmoglein (DsG1-3) and anti-skin basal protein (BP180-230) antibodies was detected.

Although serological tests for Herpes simplex viruses, Epstein-Barr virus (EBV), cytomegalovirus (CMV), varicella-Zoster virus, human immunodeficiency virus, influenza A and B-viruses, toxoplasma, hepatitis $\mathrm{B}$ and $\mathrm{C}$ viruses were negative, a quantitative polymerase chain reaction (qPCR) conducted on peripheral whole venous blood detected the presence of four Herpes virus family members, namely CMV (1165 copies $/ \mathrm{mL})$, EBV (2589 copies/mL), HHV-6 (5198 copies/mL) and HHV-8 (698 copies $/ \mathrm{mL}$ ).

Instrumental investigations further revealed the presence of two distinct bacterial infections, endocarditis and pneumonia. Methicillin-resistant Staphylococcus aureus (MRSA) was identified in serial blood cultures, and transesophageal echocardiography showed a vegetation on the ICD electrode, compatible with the diagnosis of infective endocarditis. According to the antibiogram profile, endocarditis was treated with intravenous (i.v.) teicoplanin (loading dose regimen $12 \mathrm{mg} / \mathrm{kg}$ every 12 $\mathrm{h}$ for 3 i.v. administrations, and maintenance dose regimen $12 \mathrm{mg} / \mathrm{kg}$ once-daily) for 8 weeks, which brought to a persistent resolution of the infection, based on both blood cultures and echocardiography examinations. ICD replacement was not immediately

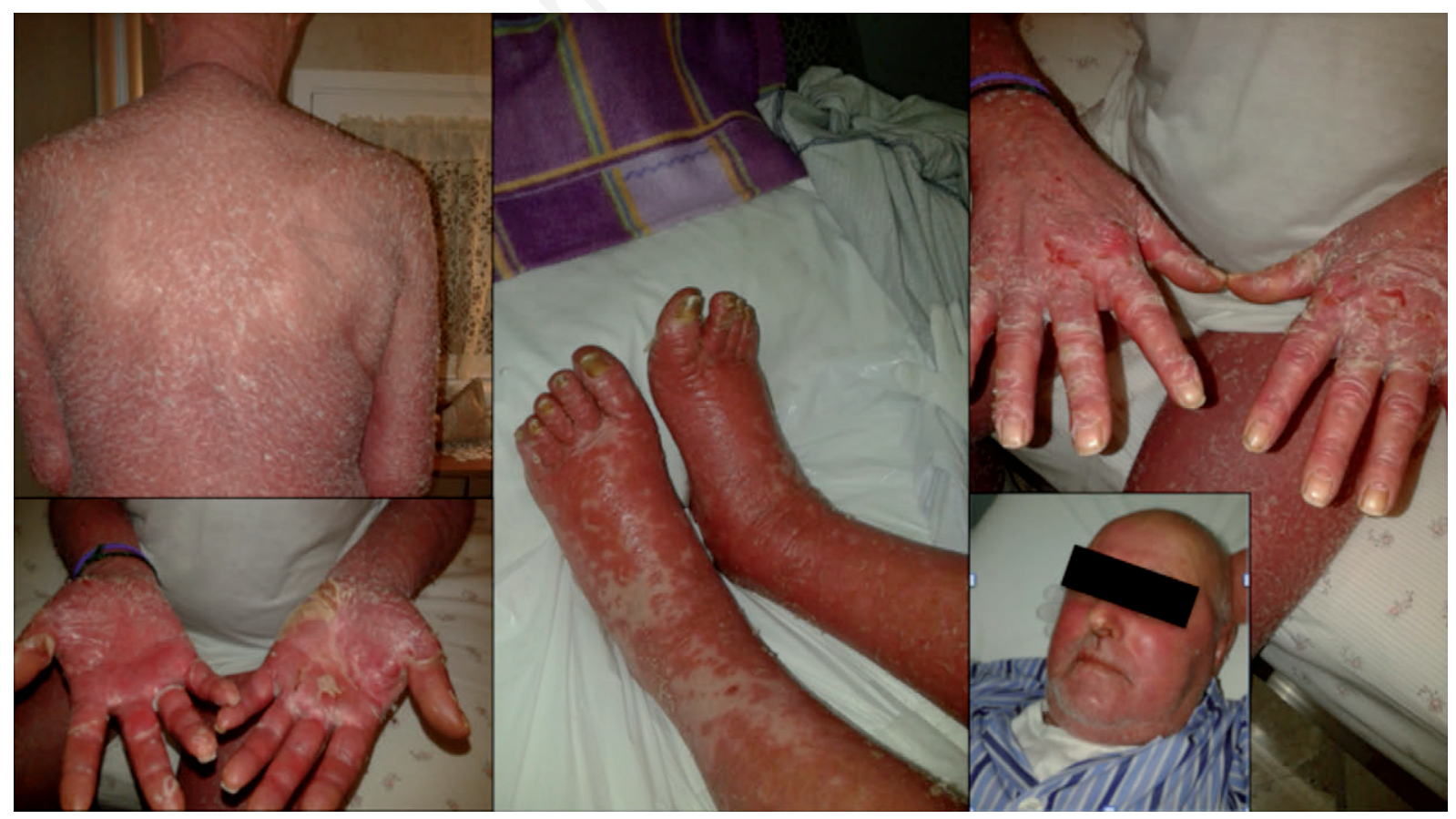

Figure 1. Patient's skin lesions (maculo-papular-desquamative erythroderma, peri-nasal and peri-oral herpes-like lesions, facial edema, and onychodystrophy) at admission to the clinic. 
performed due to the frail medical conditions of the patient during the teicoplanin therapy and to the increased risk of post-surgery complications (for the previous pulse generator pocket infection, where the new ICD would have been placed). However, despite this risk, ICD replacement was programmed as soon as the patient's clinical conditions were stable enough following teicoplanin therapy.

A thoracic computed tomography (CT) investigation, which was conducted at the same time, revealed pneumonia and confirmed multiple lymphadenopathies without splenomegaly as well as no evidence of neoplasia. A marked anti-mycoplasma pneumoniae immunoglobulin M positivity allowed validating the empirical antibiotic therapy previously started (i.v. piperacillin/tazobactam, $4.5 \mathrm{~g}$, three times a day, and i.v. levofloxacin, $500 \mathrm{mg}$, once a day). These two bacterial infections, in addition to multiple organ disfunctions and a SOFA score of $6 / 28$ points, allowed sepsis diagnosis. ${ }^{1}$

Histological and immunohistochemical examinations of skin biopsies (taken from the back, upper and lower limbs), which were performed in order to better define the severe cutaneous involvement of the disease, disclosed an immune-morphological picture that was suggestive of a dermatitis reaction to drugs (Figures 2 and 3). Cutaneous T-cell lymphoma was discarded based on the polyclonal features of T-cell receptor gamma-chain gene rearrangement obtained by molecular investigation.

Based on the diagnostic suspicion of severe adverse drug reaction, allopurinol regimen was immediately discontinued. Erythroderma and generalized edema were thus treated with systemic steroids (i.v. methylprednisolone $0.5 \mathrm{mg} / \mathrm{kg} /$ daily) and i.v. furosemide, which resulted in a complete clinical, laboratory and instrumental remission that took place in 2 weeks. Corticosteroids tapering was therefore performed (10 mg/week). However, during corticosteroids tapering while assuming oral prednisone $25 \mathrm{mg}$ daily, the patient experienced a severe relapse of cutaneous, respiratory and systemic symptoms and signs, which were associated with the onset of a serious bilateral muscular weakness affecting the lower limbs. The thoracic high resolution-CT disclosed an acute interstitial pneumonia, which was confirmed by molecular viral load quantification on the peripheral blood, showing a remarkable increase of Herpes virus DNA copies (CMV: 21,634 copies/mL, EBV: 1849 copies/mL, HHV-6: 1728 copies/mL, HHV-8: 4951 copies/mL), as well as markedly positive human CMV antigenemia. An acute demyelinating neuromyopathy was also revealed by lower limb electromyography. The complete remission of clinical, laboratory and radiological abnormalities was obtained once again, in parallel with the normalization of the qPCR-based viral load, by i.v. administration of ganciclovir ( 5 $\mathrm{mg} / \mathrm{kg}$ twice a day, 8 weeks), methylprednisolone ( 0.5 $\mathrm{mg} / \mathrm{kg} /$ daily) and of intravenous immunoglobulin (IVIG, $400 \mathrm{mg} / \mathrm{kg}$ for 5 days).

The clinical, laboratory and instrumental features observed in our patient, strongly suggested a particular case of a severe recurrent adverse reaction to allopurinol, since the observed reaction was rated as definite DRESS according to the RegiSCAR scoring system (score $>5$ ), ${ }^{2}$ or typical DRESS if using the Japanese group's criteria (score $=7 / 7$ ). ${ }^{3}$

The patient was discharged from the hospital in a good general clinical condition, while he was assuming oral methylprednisolone (20 mg, daily) and oral valganciclovir (900 mg, daily). He stopped assuming valganciclovir after 3 weeks and was administered IVIG after one month. ICD replacement, patch testing and the test for in vitro T-cell proliferation were programmed but unfortunately were never performed because three weeks after the last IVIG administration, while the patient was assuming oral methylprednisolone $8 \mathrm{mg}$ daily, a new severe cutaneous and systemic relapse took place, which lead to a rapid worsening in respiratory function and subsequent death of the patient.

\section{Discussion}

The DRESS syndrome is a severe life-threatening systemic adverse reaction induced by drugs. As results from the literature, its estimated incidence varies from 1 in 1000 to 1 in 10,000 drug exposures, ${ }^{4,5}$ it has also been included in the current nosological classification of rare diseases (ORPHA:139402). Anticonvulsants (carbamazepine 27\%), allopurinol (11\%), sulfonamides and anti-infectious drugs have been reported to be those most frequently associated with the DRESS syndrome. ${ }^{4}$

The most common scoring systems to establish the clinical diagnosis of the DRESS syndrome are the RegiSCAR ${ }^{2}$ and the Japanese group's criteria. ${ }^{3}$

The clinical manifestations of the DRESS syndrome include skin rash accompanied by fever, lymphadenopathy, and a multisystemic involvement. The typical onset is delayed, usually arising 2-6 weeks following drug exposure, with clinical manifestations persisting or aggravating despite drug discontinuation in a minority of patients. ${ }^{4}$

The severe systemic involvement of the DRESS syndrome, observed in the present case report, included fever, erythroderma, acute interstitial pneumonia, hematological, renal, cardiac, hepatic and pancreatic dysfunction accompanied by a peripheral neuromyopathy, described only by Vasanthan and coworkers. ${ }^{6}$ At admission, the patient presented sepsis due to two different bacterial infections (pneumonia and 
MRSA-endocarditis) taking place, probably as a consequence of an HHV-6-induced immunodepression ${ }^{7}$ that has rarely been reported in DRESS patients. ${ }^{8,9}$

The presence of unusual severe comorbidities and the confounding factors complicated the differential diagnosis in our case. The common DRESS differential diagnosis includes a great variety of syndromes, which are mainly infectious, autoimmune, allergic, neoplastic, and drug-induced. ${ }^{4,10}$ The Staphylococcal scalded skin syndrome or toxic shock syndrome was definitively excluded, based on the stable MRSA clearance at the time of skin relapse, during teicoplanin therapy. A negative total body-CT scan and the normal level of the neoplastic markers, allowed the exclusion of the paraneoplastic syndrome, the absence of auto-antibodies excluded autoimmune diseases and psoriasis was ruled out due to the absence of joint pain in the patient with a negative history of related skin lesions, in spite of the HLA-Cw6 risk genotype. ${ }^{11}$ Skin biopsy revealed the typical DRESS syndrome (Figures 2 and 3) and ruled out contact or atopic dermatitis and T-cell lymphoma.

The complete pathogenesis of the DRESS syndrome is far from being elucidated. Further studies should be promoted to establish the exact epidemiology, etiology and pathogenesis of the DRESS syndrome. A complex, although unclear, interplay between abnormalities in the hepatic metabolism of drugs, alterations in immunological mechanisms, reaction to Herpes viruses and genetic predisposition (HLA alleles), is responsible for DRESS pathogenesis. ${ }^{4}$ Previous reports have suggested that the systemic manifestations and severity of the DRESS syndrome are related to the reactivation of human Herpes viruses (EBV, CMV, HHV-6, HHV-7, HHV-8), ${ }^{4,12}$ in particular of HHV6. ${ }^{3,4,13}$ HHV-6 has been detected in the blood of

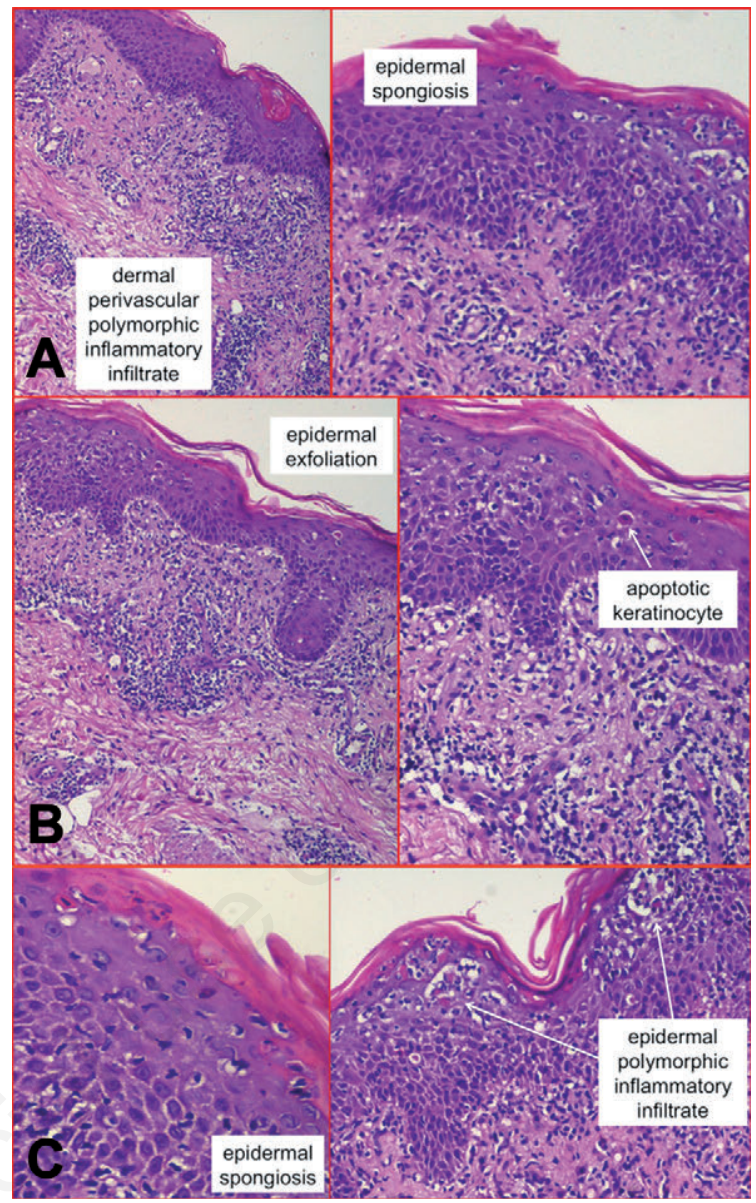

Figure 2. Hematoxylin and eosin stain of skin biopsy section. Epidermis showing spongiosis (A, B), necrotic/apoptotic keratinocytes (B) and focal lymphocytic-granulocytic epidermal exocytosis (C). The histological examinations of the skin biopsies showed a predominantly perivascular dermal inflammatory infiltrate of T-lymphocytes, plasma cells, esinophils, neutrophils (A).

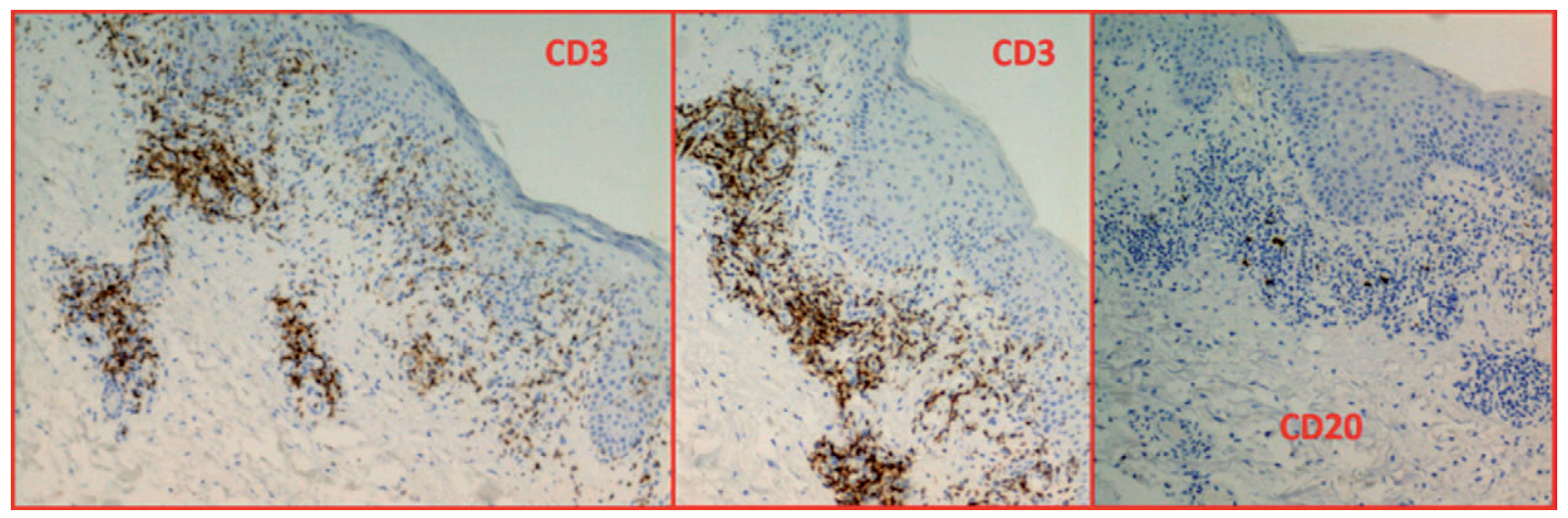

Figure 3. Immunohistochemical examination of skin biopsies. Dermal and epidermal T-lymphocytes (CD3) infiltrate is evident in parallel with the absence of B-lymphocytes (CD20). 
$60-80 \%$ of DRESS patients and, for this reason, HHV6 reactivation was included as diagnostic criteria for the drug-induced hypersensitivity syndrome by the Japanese group. ${ }^{3}$ Although HHV-6 reactivation might be useful in diagnosis, its role in the DRESS pathogenesis remains unclear. Flamand et al. observed that HHV-6 was reported to have immunomodulating effects in vitro. HHV-6 can induce the production of interleukin-1 $\beta$ and tumor necrosis factor- $\alpha$, which suppresses the function of T-lymphocytes, due to reduced interleukin- 2 production, and suppresses bone marrow by inducing interferon- $\alpha{ }^{7,12}$

A remarkable reactivation of multiple human Herpes viruses (HHV-6, CMV, HHV-8, EBV) was observed in our case, in particular of HHV-6 at admission (5198 copies $/ \mathrm{mL}$ ) and of CMV during relapse $(21,634$ copies $/ \mathrm{mL}$ ). Komura et al. described an active CMV infection after HHV-6 reactivation. ${ }^{12}$ As previously reported, isolation of HHV-6 after organ transplant occurs within the first four weeks in contrast with CMV infection, which typically occurs 4-16 weeks after transplantation. ${ }^{12-14}$ Thus, severe CMV infection appears to be strongly associated with HHV-6 reactivation in patients receiving organ transplantation. ${ }^{12-14}$ Therefore, the immunomodulating action of HHV-6 might have induced CMV reactivation, at relapse, during steroids tapering. Despite the risk of viral infection has been shown to be elevated in patients taking steroids, ${ }^{15}$ especially following organ transplantation, ${ }^{16}$ the clinical conditions in DRESS patients have shown to improve by using systemic steroids, due to the suppression of both an excessive immune response to drugs and to the massive cytokines production induced by viruses. ${ }^{17}$ According to this observation, CMV and HHV-6 reactivation has been reported in DRESS patients immediately after steroids tapering. ${ }^{8}$ The contrast between these functions (i.e. immune response suppression and viral infection reactivation) in steroids makes steroid treatment hard in DRESS patients and a careful reduction in steroid dose is required as soon as possible to reduce the probability of relapses.

Descamps and collaborators have outlined a possible procedure to follow for DRESS management and therapy.$^{18}$ According to these authors, ${ }^{18}$ the first intervention is the immediate withdrawal of the culprit drug, which, together with the prompt administration of systemic corticosteroids considered the gold standard treatment for DRESS, can reduce the morbidity and mortality to a minimum. IVIG was administered in patients affected by DRESS syndrome, especially in non-responders to systemic steroids or when steroid treatment was contraindicated..$^{18}$ The presence of signs of a major viral reactivation promoted the use of antiviral ganciclovir. ${ }^{8}{ }^{818}$ As results from the literature, other second-line strategies can be tested in DRESS syndrome: plasmapheresis and the use of immunosuppressive drugs, such as cyclophosphamide, cyclosporine, interferons, mycophenolate mofetil, and rituximab. ${ }^{18,19}$

Despite prognosis is favorable in the majority of DRESS patients and often the syndrome regresses completely following the withdrawal of the culprit drug and with an appropriate treatment, ${ }^{4}$ our case presented multiple and severe relapses and had a fatal outcome. The estimated DRESS mortality rate is $10 \%$ mainly due to hepatic necrosis. ${ }^{4,5}$ In our patient a new Herpes virus-induced pneumonia was probably responsible for the second fatal relapse.

\section{Conclusions}

Skin rash with systemic symptoms, associated with changes in drug therapy occurring a few weeks before, should induce a strong suspicion of the DRESS syndrome. The early diagnosis of the DRESS syndrome allows the prompt suspension of the culprit drug as well as the administration of systemic steroids. The early detection of DNA Herpes viruses is recommended, both at admission and during relapses, in order to promptly expose patients to antiviral therapy, in the case of a significant reactivation of HHV-6 or CMV. The immunomodulating action of HHV-6 could have induced the bacterial co-infections (MRSA endocarditis and pneumonia), rarely described, as well as the significant CMV reactivation, at relapse, during steroids tapering.

In conclusion, although our case manifested certain features, which were typical of the DRESS syndrome, it was also an unusual diagnostic and therapeutic challenge, not only due to the rarity of the disease, but even more so due to the presence of unusual serious comorbidities, complications and confounding factors that were present in a frail patient already characterized by a severe cardiopathy.

\section{References}

1. Singer M, Deutschman CS, Seymour CW, et al. The third international consensus definition for sepsis and septic shock (Sepsis-3). JAMA 2016;315:801-10.

2. Roujeau J-C, Allanore L, Liss Y, Mockenhaupt M. Severe cutaneous adverse reactions to drugs (SCAR): definitions, diagnostic criteria, genetic predisposition. Dermatol Sinica 2009;27:203-9.

3. Shiohara T. Drug-induced hypersensitivity syndrome (DIHS): a reaction induced by a complex interplay among herpes viruses and antiviral and antidrug immune responses. Allergol Int Off J Jpn Soc Allergol 2006;55:1-8.

4. Rajagopalan M, Sarda A, Das S, Biswas P. Drug reaction with eosinophilia and systemic symptoms: an update and review of recent literature. Indian J Dermatol 2018;63:30-40. 
5. Chiou CC, Yang LC, Hung SI, et al. Clinicopathological features and prognosis of drug rash with eosinophilia and systemic symptoms: a study of 30 cases in Taiwan. J Eur Acad Dermatol Venereol 2008;22:1044-9.

6. Vasanthan T, Rajaguru G, Venkatesh C, et al. DRESS syndrome with peripheral neuropathy due to reactivation of cytomegalovirus in a child. J Glob Infect Dis 2015; 7:89-90.

7. Flamand L, Gosselin J, Stefanescu I, et al. Immunosuppressive effect of human herpesvirus 6 on T-cell functions: suppression of interleukin-2 synthesis and cell proliferation. Blood 1995;85:1263-71.

8. Arakawa M, Kakuto Y, Ichikawa K, et al. Allopurinol hypersensitivity syndrome associated with systemic cytomegalovirus infection and systemic bacteremia. Intern Med 2001;40:331-5.

9. Della-Torre E, Yacoub MR, Pignatti P, et al. Optimal management of DRESS syndrome in course of infectious endocarditis. Ann Allergy Asthma Immunol 2013;110:303-5.

10. Grant-Kels JM. Exfoliative dermatitis. In: Burns T, Breathnach S, Cox N, Griffiths C, eds. Rook's textbook of dermatology. 8th Edition. New York: WileyBlackwell; 2010.

11. Chen L, Tsai TF. HLA-Cw6 and psoriasis. Br J Dermatol 2018;178:854-62.

12. Komura K, Hasegawa M, Hamaguchi Y, et al. Druginduced hypersensitivity syndrome associated with human herpesvirus 6 and cytomegalovirus reactivation. J Dermatol 2005;32:976-81.

13. Kano Y, Hiraharas K, Sakuma K, Shiohara T. Several herpesviruses can reactivate in a severe drug-induced multiorgan reaction in the same sequential order as in graft-versus-host disease. Br J Dermatol 2006;155:301-6.

14. Herbein G, Strasswimmer J, Altieri M, et al. Longitudinal study of human herpesvirus 6 infection in organ transplant recipients. Clin Infect Dis 1996;22:171-3.

15. Stuck AE, Minder CE, Frey FJ, et al. Risk of infectious complications in patients taking glucocorticosteroids. Rev Infect Dis 1989;11:954-63.

16. Emery VC, Cope AV, Sabin CA, et al. Relationship between IgM antibody to human cytomegalovirus, virus load, donor and recipient serostatus, and administration of methylprednisolone as risk factors for cytomegalovirus disease after liver transplantation. $J$ Infect Dis 2000;182:1610-5.

17. Tohyama M, Yahata Y, Yasukawa M, et al. Severe hypersensitivity syndrome due to sulfasalazine associated with reactivation of human herpesvirus 6 . Arch Dermatol 1998;134:1113-7.

18. Descamps V, Ben Saïd B, Sassolas B, et al. Management of drug reaction with eosinophilia and systemic symptoms (DRESS). Ann Dermatol Venereol 2010; 137:703-8.

19. Husain Z, Reddy BY, Schwartz RA. DRESS syndrome: Part II. Management and therapeutics. J Am Acad Dermatol 2013;68:709.e1-9. 